\title{
Editorial
}

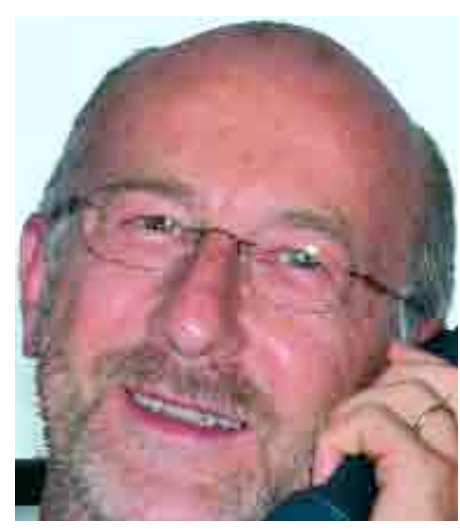

\section{Integrative Medizin}

Unser bestehendes Medizinsystem erf reut sich einer enormen technischen Aufrüstung. Neue diagnostische und therapeutische Verfahren werden für hohe Summen entwickelt und in das Vergütungssystem der Krankenkassen integriert. Neue und, so erfährt man, bessere Wirksubstanzen werden mit wachsendem Druck immer schneller in heiss umkämpfte Märkte gepresst. So sollte man meinen, die Menschen würden immer gesünder. Doch weit gefehlt, das Gegenteil ist der Fall: Die Zahl der chronisch Kranken steigt fast linear mit den Kosten im Gesundheitswesen an, und trotz allen medizinischen Fortschritts ist in der Schulmedizin gegen die Leiden gerade dieser PatientInnen offensichtlich «kein Kraut gewachsen». Erfolgreiche Hilfe bieten hier in vielen Fällen komplementärmedizinische Heilverfahren. Schul- und Komplementärmedizin gehören zusammen, nur zusammen sind sie ein Ganzes, ergänzen sich zum Optimum, und nur so können sie der Vielfalt der Patientenbedürfnisse gerecht werden. Schul- und Komplementärmedizin als integraler Bestandteil einer Medizin, die Tradition und Moderne gleichwertig vereint, eine Empirie- und Evidenz-basierte Medizin, eine integrative Medizin also.

All dem tragen wir Rechnung im jetzt erweiterten Titel und Inhalt unserer Fachzeitschrift. Schwerpunkte liegen bei Themen der Komplementärmedizin, ergänzt durch wichtige und aktuelle Beiträge aus dem Bereich der Ernährungsmedizin und Gesundheitsprävention. Gesundheitspolitische Themen werden ebenfalls ein Schwerpunkt sein. Zentraler Bestandteil werden künftig einem «Peer Review» unterzogene Beiträge sein wie z.B. wissenschaftliche Forschungsarbeiten, klinische Studien, Reviews und Kasuistiken. Und für richtungsübergreifende fachliche Diskussionen stellen wir reichlich Raum zur Verfügung. Im redaktionellen Fokus mit nationalem und internationalem Bezug stehen aktuelle wissenschaftliche Erkenntnisse, zukunftsträchtige Strömungen und vor allem die vielfältigen Möglichkeiten der praktischen Anwendung einer Medizin, die den Patienten aktiv an seiner Gesundung und Gesunderhaltung beteiligen.

Die «Schweizerische Zeitschrift für Ganzheitsmedizin» erscheint mittlerweile im 17. Jahrgang, sie hat sich in dieser Zeit in Fachkreisen nicht nur der Schweiz etabliert. Sie hat sich unter anderem zum Ziel gesetzt, auf einer fachlich-fundierten und sachlichen Basis das zu vermitteln, was eingefleischte Gegner der Komplementärmedizin vehement leugnen: Komplementärmedizin ist wissenschaftlich begründet, wirksam und kostengünstig, sie ist eine sinnvolle Bereicherung des bestehenden Medizinsystems. Dies zeigt übrigens auch die soeben abgeschlossene PEK-Studie (Projekt Evaluation Komplementärmedizin) in ganz eindeutiger Weise: komplemetärmedizinisch tätige Ärzte - im Vergleich zu ihren rein schulmedizinisch ausgerichteten KollegInnen - behandeln ihre PatientInnen nicht nur erfolgreicher, sondern auch wesentlich kostengünstiger.

Die «Schweizerische Zeitschrift für Ganzheitsmedizin» erhielt jetzt, neben dem stetig wachsenden Wissenschaftlichen Beirat, eine Schriftleitung, die sich aus den Experten Prof. Dr. med. Reinhard Saller/UniSpital Zürich, Univ. Doz. Dr. med. Peter Heusser/Uni Bern und Dr. med. Jörg Fritschi/Präsident der UNION, zusammensetzt. Der Kreis zwischen universitär-wissenschaftlich repräsentierter und breit in der Praxis umgesetzter ärztlicher Komplementärmedizin ist damit geschlossen.

Dr.rer.nat. Stefan R. Becker, Herausgeber 